\title{
FUNDAMENTAL SOLUTIONS TO SOME ELLIPTIC EQUATIONS WITH DISCONTINUOUS SENIOR COEFFICIENTS AND AN INEQUALITY FOR THESE SOLUTIONS
}

\author{
A. G. RAMM
}

Abstract. Let $L u:=\nabla \cdot(a(x) \nabla u)=-\delta(x-y)$ in $\mathbb{R}^{3}, 0<c_{1} \leqslant a(x) \leqslant c_{2}, a(x)$ is a piecewise-smooth function with the discontinuity surface $S$ which is smooth. It is proved that in an neighborhood of $S$ the behavior of the function $u$ is given by the formula:

$$
u(x, y)= \begin{cases}\left(4 \pi a_{+}\right)^{-1}\left[r_{x y}^{-1}+b R^{-1}\right], & y_{3}>0, \\ \left(4 \pi a_{-}\right)^{-1}\left[r_{x y}^{-1}-b R^{-1}\right], & y_{3}<0 .\end{cases}
$$

Here the local coordinate system is chosen in which the origin lies on $S$, the plane $x_{3}=0$ is tangent to $S, a_{+}\left(a_{-}\right)$is the limiting value of $a(x)$ on $S$ from the half-space $x_{3}>0$, $\left(x_{3}<0\right), \quad r_{x y}:=|x-y|, R:=\sqrt{\rho^{2}+\left(\left|x_{3}\right|+\left|y_{3}\right|\right)^{2}}, \rho:=\sqrt{\left(x_{1}-y_{1}\right)^{2}+\left(x_{2}-y_{2}\right)^{2}}$, $b:=\left(a_{+}-a_{-}\right) /\left(a_{+}+a_{-}\right)$. If $S$ is the plane $x_{3}=0$ and $a(x)=a_{+}$in $x_{3}>0, a(x)=a_{-}$ in $x_{3}<0$, then $(*)$ is the global formula for $u$ in $\mathbb{R}^{3}$. Inequality for the fundamental solution for small and large $|x-y|$ follows from formula $(*)$.

Mathematics subject classification (1991): 35R30. problems

Key words and phrases: Fundamental solutions, elliptic equations, discontinuous coefficients, inverse

\section{REFERENCES}

[K] KozLov, S., Asymptotics of the fundamental solutions of differential equations of second order, Matem. Sbornik 113 no. N2 (1980), 302-323, (Russian).

[LSW] LitTman, W., Stampalchia, G., WeinBerger, H., Regular points for elliptic equations with discontinuous coefficients, Ann. Scuda Norm. Super. Pisa 17 (1963), 43-77.

[R] Ramm, A. G., Multidimensional Inverse Scattering Problems, Longman/Wiley, New York, 1992 , pp. 1-496, expanded Russian edition, MIR, Moscow, 1994, pp. 1-496. 